\title{
SOCIO-ECONOMIC ASPECTS OF THE INTERACTION OF SOCIAL AUTHORITY DURING THE COVID-19 PANDEMIC IN THE CONTEXT OF PUBLIC HEALTH OF THE POPULATION
}

\author{
Oleg Chornyi'
}

\begin{abstract}
The purpose of the study is to substantiate the transformation of approaches to the formation of the latest system of socio-economic factors of public health to combat the COVID-19 pandemic at the present stage. The features of the economic aspects of public health policy and possible directions of its modern transformation have been studied. The urgency of public perception of the need to implement reform processes in the healthcare system at the stage of countering the coronavirus pandemic has been substantiated. The main measures for reforming the state policy of the healthcare system in the European countries are analyzed. The main functions of state policy in relation to the processes of economic provision of the healthcare system in developed countries have been investigated. The subject of the study is the economic factors of public-government cooperation in reforming the healthcare system during the COVID-19 pandemic. Research methods. The methodological basis of the study was a systematic approach, which allowed to consider the control system and managed subsystems as a holistic complex of medical and social services to the population during the COVID-19 pandemic. To ensure the completeness of the coverage of the subject of research, its conditional structuring on an empirical and theoretical level was carried out, as well as various methods of scientific research were used. At the empirical level, methods of statistics and semantic analysis were used to reveal the specifics of the object of study, as well as to study the impact of socio-economic factors on policy development to combat COVID-19, study the scientific literature on the experience of medical care for patients with COVID-19 in foreign countries. Conclusions. Today, the state of the healthcare system is characterized by a stage of crisis, and the healthcare sector is not a high priority in the state budget policy, and, consequently, the issue of funding is becoming more acute. The transformation of the healthcare system should consist in the organization of the provision of medical care available to the population of the country on the basis of fundamental transformations of financing, with the involvement of financial resources other than state. It is the interaction and close cooperation between the state and business that can contribute to the development of new forms and models of social-government interaction to improve the quality of life in society. Attracting private business, small, medium, and large market participants will be able to bring new solutions to change the socio-economic factors of healthcare.
\end{abstract}

Key words: public policy, economic aspects, European countries, pandemic, reforms, health protection, counteraction.

JEL Classification: L43, R20, I18, I12, H51

\section{Introduction}

Measures of transformation of the healthcare system implemented in recent years are, in fact, focused primarily on reducing the gap with Western European countries in the volume of hightech healthcare, the level of technical equipment of medical institutions as medical care to patients. The main tool is to increase funding for the healthcare system. Therefore, maintenance of the improvement of technical equipment of medical institutions and increasing funding for their activities, as a priority, will only reduce the gap with European countries in mortality rates, but not overcome it, and will have a relatively weak impact on morbidity.

Public opinion and the participation of civil society should not be restricted or abolished when public authorities are curtailed, unless the measures themselves create a high risk of the

\footnotetext{
Corresponding author:

${ }^{1}$ Interregional Academy of Personnel Management, Ukraine.

E-mail: chornyioleg86@gmail.com

ORCID: https://orcid.org/0000-0001-6476-5158
} 
disease spreading. Highly motivated societies are key factors in effective action, and civil society can monitor responses in the field, note how these interventions affect vulnerable groups, and report problems to public authorities. To do this, they must have access to reliable information, and also to officials, to have an open dialogue with them and to report on existing problems.

It is important to ensure the economic feasibility of approving standards and the gradual implementation of them for groups of diseases with adequate funding. The planned changes in the system of compulsory health insurance will increase the availability and quality of medical care, but are insufficient to overcome structural imbalances in the healthcare system, improve coordination of its units, the formation of strong motivation of insurance medical organizations and medical institutions to improve the quality of treatment and prevention work and more efficient use of industry resources. However, it should be noted that the problem of differentiating free and paid healthcare cannot be solved in the next few years due to economic and political constraints.

When formulating and implementing public policy, it is necessary to ensure that society has the necessary information to protect themselves and help others, and the channels of feedback from society should be always open. Community leaders should be involved in dissemination of information, provide access to information and freedom of speech, and people should be able to protect their health and seek help if it is necessary. Healthcare workers also need timely and adequate information to act quickly and effectively, and its dissemination can be ensured through civil society institutions.

\section{Analysis of recent research}

Studies show that despite the obvious positive development, the measures taken are not enough to address the problems that have accumulated: the predominant focus is on treating diseases rather than preventing them, weak coordination of measures for the development of the medical sector with efforts to improve non-medical health promotion factors, failure to pay significant attention to the potential of these factors, insufficient and sharply differentiated actual unavailability of quality medical care, weak protection of patients' rights, risks of excessive forced costs for treatment with its formal free of charge, lack of appropriate conditions and incentives for the most efficient use of healthcare resources.

Public authorities must refrain from restricting freedom of speech and access to information and ensure that the public is regularly able to receive complete and up-to-date information, and take measures to prevent and cessation of discrimination. The way public authorities and the media talk about the epidemic, the ways in which the disease is transmitted, the people who suffer from COVID-19, form an idea of how these people and communities are perceived and treated by other people. This can lead to significant opposition to certain groups of the population, contribute to discriminatory treatment, forced additional isolation of people or violation of their privacy, affecting both mental health and access to various services.

Within some epidemic, discrimination in the provision of medical care, also denial of treatment due to nationality, country of origin, lack of insurance, socio-economic or other status, not only deprives a person of necessary medical services, but also endangers the health of others people and reduce the effectiveness of measures in general. World experience shows that private medicine in most countries is able to effectively perform the tasks of public health (Hick et al., 2020). Precisely private medicine, which has the resources and experience, it is an effective resource-saving tool for the state. In order to improve the quality of medical care for insured citizens, it is necessary to ensure a competitive environment between medical organizations.

It should also be taken into account that there is an opinion formulated by a number of experts that for the progressive development of the national healthcare system it is necessary to look for new solutions in the organization of medical institutions. Equipping of medical institutions with new medical equipment is an insufficient condition for the development of the healthcare system, but it is certainly important at the present stage for the state humanitarian policy. These facts go beyond the responsibility of individual agencies and involve fundamental changes in the resource base of healthcare and the behavior of all its subjects.

Appropriate measures should be addressed to the entire population of the country, taking into account features of specific groups. The necessary changes must be made, of course, not to the detriment of the further development of medical care. In turn, it needs significant technical re-equipping and 
involvement with talented, ambitious young people, aimed at constant creative growth and mastery of innovation. The development of informational technology will have a growing impact on the formation of a new healthcare system.

It is important to create a system of training of non-medical healthcare professionals, employees of executive bodies in charge of the humanitarian sphere, managers of institutions, specialists in information support in healthcare. These specialists can become a leader of ideas for the formation of a healthy lifestyle and disease prevention. This task is possible through "public health schools", which have experience in many countries, and begins to form on the basis of several medical universities. For large-scale implementation of the proposed approach, permission for medical universities to conduct postgraduate training of specialists who do not have medical education, but are involved in healthcare, is required.

Mandatory restrictive measures do not help to remove barriers for the most vulnerable groups; on the contrary, they increase these barriers and potentially increase people's vulnerability. Such measures can undermine trust between public authorities and society, destroy people's sense of responsibility for their lives, when it is so necessary to continue to take care of themselves and others. All these approaches are not new, they are the basis of public health, providing the conditions of proportionality and necessity of services provided, coverage of the most vulnerable groups.

Any restriction must pursue a specific purpose and be necessary, effective and based on timelimited evidence. State social and humanitarian policy should provide for direct and significant participation of society in the activities of all public authorities, which provides for open information and transparency in making decision. In this case, the relevant measures will meet the real needs and requirements of people, improve trust in government, mutual trust in society. Civil society has a key role in helping people seek medical care in a timely manner, adhere to self-isolation, receive the necessary medication, and care for each other's families.

\section{Healthcare reform processes in the response to the coronavirus pandemic}

Planned retaliatory measures are effective only when society itself takes an active part in their development, implementation and monitoring.
Public authorities should ensure that, when developing some measures, including travel restrictions or testing regimes, society participates in the development of measures, not only at the initial stage, but also at all subsequent stages of response. The participation of civil society as a whole is also necessary for people to perceive the programs as they really need and to trust these activities.

All high-risk groups need to be identified to ensure their equal participation in the decisionmaking process, and if society is not yet involved in decision-making, this needs to be made a priority. Public authorities should properly prepare budgetary healthcare facilities, provide disease detection, prevention and treatment. Epidemiological surveillance is required to detect, isolate, test, and treat each patient, to break the chain of transmission, to prevent and stop the further spread of the infection. It is necessary to identify and isolate as many as possible patients and quarantine those who have been in close contact with them (Liu et al., 2020). Even if it is not possible to stop the spread of the disease, it can be slowed down and, thus, protect medical institutions, nursing homes and other important facilities.

One of the most important and effective responses from the public health system is to actively inform the public about what is known and unknown at the moment, what is being done to obtain information what is done to save lives and minimize the adverse effects of the epidemic. Active systematic communication and interaction of public authorities with the population helps to cope with general confusion and avoid misunderstandings in order to assess the risks to their own health and the health of their loved ones.

Public authorities must take measures to protect health workers from all forms of discrimination and provide them with the necessary support, they should talk about the epidemic together with health workers. Everyone, regardless of race, nationality, gender and profession, must be sure that physicians and government agencies observe the utmost care and confidentiality in the handling of their personal data. However, the abuse of sanctions often has serious negative consequences for a particular person and does not take into account the realities of people's lives.

In emergencies that threaten the health of the population, sanctions often have a broad and vague 
interpretation, and their application risks being arbitrary, and people against whom these sanctions are applied are often members of the most vulnerable communities. A much greater effect can be achieved by encouraging society to protect people by providing them with everything they need, and the existing inequality means that some groups may be more affected by the epidemic. Both the virus and the corresponding actions of public authorities can be a cause of additional discrimination, the epidemic often reveals the existing inequality in society, when already vulnerable people suffer the most from the epidemic.

People who do not have the money for medical care are more likely to refuse virus testing, prisoners will be virtually unable to control their access to medical services; without investing significant additional resources, it is impossible to organize hand washing for people in settlements or communities where there is no running water and the burden of caring for family members falls mainly on women. Public authorities should strive to ensure that public health policies, from infrastructure to affordable testing services and inpatient care, are provided with the resources needed for prevention, treatment and containment of the epidemic.

The responsibilities of states in respecting the right to health of citizens do not include only the organization of accessible, affordable and quality health services and the dissemination of information, but also the creation of health infrastructure provided with everything necessary to meet society's health needs including in the prevention, treatment and control of epidemics. States must build health capacity, taking into account existing domestic and international resources, to make reliable use of the scientific advances and testing that are available and that are physically and financially available to someone in need. This allows a person to take measures to protect their own health and well-being, the health and well-being of their loved ones as well.

Success in fighting the epidemic depends on testing people at the highest risk of infection. It is unacceptable that the obstacle to testing should be the need to pay for testing at their own expense, testing should be free or at least offered at a price affordable to all. With limited resources, access to testing and care should be vulnerability and need, not people's financial means. It is necessary to provide all health workers with information on the priority of coverage of the most vulnerable people, which include low-income citizens, people with chronic diseases, people with disabilities (Fasano et al., 2020).

It is important to remember that health workers are also a vulnerable group, and that their safety and protection must also be a priority. Diagnosis and medical care for COVID-19 should be accessible to all and, most importantly, they should be of high quality, vulnerable groups should be identified and their services should be purposefully covered. Effective treatment and prevention for all groups of the population is possible only if medical care is provided where it is convenient for people.

Public authorities must work with communities to find solutions for people who cannot access these services through quarantine, it must be provided, and health professionals must be able to provide them without risking being infected with COVID-19. The private sector is investing more and more in measures against the COVID-19 epidemic, and public authorities must ensure that progress is not reversed or reverted through the redistribution of resources.

Funding of the fighting with the global epidemic should not come from other health sectors, and every effort should be made to find and raise funds outside existing budgets. There is a need to ensure continued access to health services and medicines, including through the provision of long-term prescriptions, and to continue funding and allocating resources to other health services, especially for the most vulnerable. The lack of diagnostics underscores the urgent need to share knowledge, information and resources in order to provide all countries with quality COVID-19 tests as soon as possible. We need to work together, sharing knowledge and resources and taking coordinated action to ensure an effective response to the current epidemic and to prevent it from recurring.

\section{Public policy of the healthcare system in the European countries}

Appropriate measures can have serious negative consequences for people in such fields as work, livelihoods, access to food and basic services. The requirement of self-isolation or closure of enterprises is a risk of losing earnings and jobs, and people working part-time and unpaid sick leave are at particular risk. These circumstances disproportionately affect groups determined by 
gender and race, socio-economic status, and the fear of losing a job are often the reasons for refusing testing, treatment or, as in the case of COVID-19, self-isolation.

Public authorities have a duty to protect people from losing their jobs, income or livelihoods through a reliable social security and insurance system. This gives people the opportunity to take care of their health and well-being, to increase the effectiveness of measures in response to the epidemic. Measures should be taken to help to support not only those who are in isolation or quarantine, but also those who are forced to stay at home due to appropriate measures. It is necessary to make sure that people can isolate themselves or take care of others without losing their income or work. If this can deprive people of food, medicine or shelter, appropriate measures must be taken to remedy such situations.

Careful consideration should be given to travel bans. In some cases, such measures are disproportionate or have limited effect. They do not always prevent the spread of the epidemic. Of course, travel restrictions can in some cases be individual, but a greater effect in preventing the spread can certainly be achieved by encouraging people to adhere to a regime of self-isolation, to be tested and to monitor contacts. In the situation with COVID-19, voluntary measures aimed at social distancing and limiting physical interaction between people effectively reduce the rate of disease spread.

Coercive measures of social distancing and social exclusion, if they are deemed necessary, proportionate and justified, must take into account the likely negative consequences for people and society so that they can be adapted to mitigate them. Travel bans or restrictions can only be imposed as a last measure, they must have a limited duration, legal justification, be verifiable and apply without discrimination.

To adequately respond to the challenges of the times, it is necessary to clarify the goals, change the priorities in health policy and carry out profound structural changes in it. The target for health development is not mortality and life expectancy, as is currently the case, but the "life expectancy adjusted for health problems" indicator used by the World Health Organization and more fully state of health of the population. However, attention to measures to promote a healthy lifestyle is inadequate for their role in the possible reduction of morbidity and mortality, compared with the actual medical care (Steier, Moxham, 2020). At the same time, opportunities for rational attraction of personal funds of the population are limited, and the development of the market of paid medical services is not accompanied by the necessary development of its regulation in the interests of consumer protection and effective competition.

Existing medical equipment is often used with low efficiency. One of the main reasons for this is the lack of adequately trained staff. The same problem was encountered in organizing the work of new medical centers built on the periphery. In foreign countries, it is generally accepted that only a high social and economic status of a doctor can guarantee the medical staffing with people with the abilities and responsibilities necessary for the full performance of professional duties. We generally do not meet this requirement.

The healthcare system is characterized by serious structural imbalances: excessive load on inpatient care with relatively weak primary development, rehabilitation, medical and social assistance; the share of inpatient care costs in total healthcare costs is almost twice the share of outpatient care, while in Western European countries they are approximately equal; insufficient coordination of medical institutions and doctors at different stages of medical care. More than a third of hospitalizations can be transferred to the outpatient stage without harm to the patient's health.

An effective transformation of the healthcare system should be that resources are allocated according to the comparative contribution of its members to improving public health indicators, and all its subjects are interested in providing the greatest social and medical effect per unit cost [Jones, 2020]. This scenario assumes that significant improvements in the health of the population must be achieved, first of all, not at the expense of the most expensive and technically difficult methods of treatment and at the expense of prevention of diseases and increase of efficiency and quality of work of medical and preventive institutions.

It will be possible to have more active contacts of doctors of different specialties to combine individual episodes of treatment, regardless of the location of doctors and patients. The possibility of monitoring the patient's condition will be significantly expanded up to tracking the impact of food quality and other factors on the course of the disease. Significantly increase the importance 
of information technology in the administrative and economic process - to increase the efficiency of the medical structure, the reorganization of outpatient care; restructuring of inpatient care; formation of a quality management system for medical care; development of health insurance institutions; development of the medical services market; increasing the efficiency of the process of introducing new medical technologies (Shangguan et al., 2020).

As mechanisms that ensure the movement towards an integrated, transparent, efficient healthcare system, we need to consider: radical expansion of the set of regulatory, economic and informational measures used to support and promote a healthy lifestyle; development of medical education; transition to an effective contract with health professionals; a clear division of responsibilities for healthcare financing between the state, the population and employers.

Measures are also needed to develop medical education, bring medical educational standards in line with the requirements of modernization of practical healthcare, in particular, doctors train to work in outpatient clinics more purposefully. The formation of a new model of postgraduate education should include: the organization of postgraduate education in the form of continuing education using a system of credit and the right to choose courses; change in the procedure for financing postgraduate education - to transfer the appropriate funds to medical institutions for the intended use and payment from these funds for the order of services for postgraduate education.

It is proposed to ensure the development of nursing medical services in the field of patient care, rehabilitation and prevention. It is expedient to transfer to nursing staff a part of functions on a prescription of medicines to chronic patients, registration of medical documents which are carried out now by doctors. It is necessary to increase the degree of differentiation of the composition of paramedics, the allocation of several groups depending on the composition and complexity of the performed functions. It is necessary to improve the unified state information system in healthcare, including the system of electronic registration for a doctor's appointment, electronic medical record, a single repository of medical data. This is included in the number of tasks to be solved within the framework of territorial programs of healthcare transformation.

\section{Socio-economic support of the healthcare system in developed countries}

In the outpatient sector, it is necessary to stimulate the development of public and private partnership, to actively involve small and mediumsized businesses in the system of providing free medical care to citizens. Currently, the main amounts of medical care are provided by public health institutions. Despite the expansion of the scope of private clinics, the list of their services is limited. At the same time, the private healthcare system is an integral part of healthcare, the potential of which is not adequately demanded by the state. However, in order to implement the set tasks, there is an urgent need to attract additional funding that can be provided by the private sector.

It is the private sector that can add more flexibility to the national healthcare system, especially in the organization of institutions. In turn, the development of the pharmaceutical industry is also moving extremely fast and the states of all countries are finding it increasingly difficult to provide the population with innovative medicines. However, such medicine is extremely expensive, so that the state budget alone is not enough to provide it. It is the interaction and close cooperation between the state and business that can contribute to the development of new forms and models of cooperation between the state and business to improve the quality of life of patients. And the involvement of the private sector will be able to bring new solutions for changes in the organization of healthcare, also in provision.

The above problems and limitations hinder the development of competition in healthcare. The main restrictions on competition are related to the specific features of health services, which are socially significant, the volume of which does not depend directly on the level of solvency of the population. Transformation of the healthcare system should consist in the organization of medical care available to the population of the country, on the basis of fundamental transformation of financing, involving other than state, financial resources, transformation of legal bases of the branch directed on democratization of management, introduction of modern medical technologies, development competition based on the patient's free choice of treatment and prevention facility and doctor.

Today, the state of the healthcare system is characterized by a stage of crisis, and the healthcare sector is not a high priority in the state budget 
policy, and, consequently, the issue of funding is becoming more acute. It is important to understand that the legislation does not specify which medical services are provided free of charge, and thus it is assumed that this list includes medical services that the consumer receives in public health facilities. The population was faced with a process when the quality of free medical care began to decline sharply, as a result of which the market for paid medical services began to grow.

This negative perception of paid medical services persists to this day, and many experts believe that medical services should be free for all categories of citizens. However, we are currently observing an active trend towards increasing the number of the private health sector and its participation in the state guarantee program. Combining the efforts of the state, business and society is becoming necessary to ensure and improve the quality of medical services, the quality of which is becoming lower every year. Today in the healthcare system the development of infrastructure is defined as a priority.

However, this is not the only problem faced by the health sector: a sharp reduction in highly qualified specialists in the field of medicine and personnel in the field of management of medical institutions; lack of access to innovative treatment of patients; lag of medicine consumption from the level of developed countries; impossibility to receive certain types of services free of charge. It is also important to develop a network of medical and obstetric centers and outpatient clinics in small towns, disease prevention and diagnosis as a means of reducing mortality in working age, development and implementation of a general scientific program to combat cancer.

The reform aims to create conditions to reduce mortality and increase life expectancy by ensuring coverage of all levels of citizens with preventive medical examinations at least once a year, motivating the population and leading a healthy lifestyle, increasing public satisfaction with the quality of health services, creating conditions for any - what citizen of the guaranteed volume of medical care, increase of attractiveness, level of qualification and prestige of a profession of the doctor as the expert in the business (Wheeler et al., 2015).

It should be noted that the target indicators for improving the activities in the system of medical services are also to ensure optimal accessibility for the population of medical organizations that provide primary healthcare; optimization of the work of medical organizations that provide primary healthcare, reduction of waiting time in the queue for citizens to apply to these medical organizations, simplification of the procedure for registration with a doctor, ensuring the timeliness of medical care to citizens living in inaccessible areas.

\section{Conclusions}

The introduction of the new technologies will stimulate structural changes in the system of healthcare, increasing the needs of the population in the new medical services and at the same time increasing expectations for the state to ensure their availability. However, all this is effectively implemented under the conditions of adequate transformation of the process of innovation development, which includes the definition of funding sources, rational organization of the order of access to the new opportunities in a period when they are in short supply. The existing model of medical care is not adapted to this and should be completed taking into account challenges of the time in accordance with peculiarities of the period of constant, intensive and expensive innovations.

Society is facing a situation that is unpredictable and rapidly evolving, the answer to this situation should not be fear, but solidarity, support, and strengthening the role of society, it is necessary to build a culture of mutual assistance, trust and kindness. In response to the COVID-19 pandemic, the realities of people's lives must be taken into account, focusing on removing barriers to protecting themselves and their communities: fear of losing livelihood, food shortages and loss of respect for their community.

When a state of emergency is declared in the field of healthcare, the powers of the executive authorities are significantly expanded, and any action or inaction of the authorities that affects the rights of citizens is subject to verification by law enforcement agencies. Observance of this principle is especially important in emergencies, society should be able to challenge the legality of actions of public authorities, if such actions are above the law, they are discriminatory, or not actually justified. Not bans, but the protection and strengthening of society, truthful information will lead to the fact that measures taken in response to the epidemic will be more effective, sustainable and humane. 


\section{References:}

Fasano, A., Antonini, A., Katzenschlager, R., Krack, P., Odin, P., Evans, A. H., ... \& Merello, M. (2020). Management of Advanced Therapies in Parkinson's Disease Patients in times of Humanitarian crisis: the COVID-19 experience. Movement Disorders Clinical Practice, 7(4), 361-372. doi: 10.1002/mdc3.12965

Hick, J. L., Hanfling, D., Wynia, M. K., \& Pavia, A. T. (2020). Duty to plan: health care, crisis standards of care, and novel coronavirus SARS-CoV-2. NAM Perspectives. Discussion paper. National Academy of Medicine. Washington, DC. doi: 10.31478/202003b

Jones, D. S. (2020). History in a crisis-lessons for CEmergency Response to COVID-19 in Canada: Platform Development and Implementation for eHealth in Crisis Management. JMIR Public Health and Surveillance, 6(2), e18995.

Liu, Q. Luo, D., Haase, J. E., Guo, Q. Wang, X. Q. Liu, S., ... \& Yang, B. X. (2020). The experiences of health-care providers during the COVID-19 crisis in China: a qualitative study. The Lancet Global Health, 8(6), e790-e798. doi: 10.1016/S2214-109X(20)30204-7

Shangguan, Z., Wang, M. Y., \& Sun, W. (2020). What caused the outbreak of COVID-19 in China: From the perspective of crisis management. International Journal of Environmental Research and Public Health, 17(9), 3279. doi: 10.3390/ijerph17093279

Steier, J., \& Moxham, J. (2020). The load and capacity model of healthcare delivery: considerations for the crisis management of the COVID-19 pandemic. Journal of Thoracic Disease, 12(6), 3022. doi: $10.21037 \% 2 F j t d-2020-054$

Wheeler, C., Lloyd-Evans, B., Churchard, A., Fitzgerald, C., Fullarton, K., Mosse, L., ... \& Johnson, S. (2015). Implementation of the Crisis Resolution Team model in adult mental health settings: a systematic review. BMC psychiatry, 15(1), 74. doi: 10.1186/s12888-015-0441-x 